\title{
Association between depression and hospital outcomes among older men
}

\author{
A. Matthew Prina MPhil, Martijn Huisman PhD, Bu B. Yeap PhD, Graeme J. Hankey MD, Leon Flicker PhD, \\ Carol Brayne PhD, Osvaldo P. Almeida PhD
}

\begin{abstract}
Background: Studies that have investigated the relation between depression and the type, nature, extent and outcome of general hospital admissions have been limited by their retrospective designs and focus on specific clinical populations. We explored this relation prospectively in a large, community-based sample of older men.
\end{abstract}

Methods: A cohort of 5411 men aged 69 years and older enrolled in the Health in Men Study was assessed at baseline for depressive symptoms, defined as a score of 7 or higher on the 15-item Geriatric Depression Scale. Participants were followed for 2 years for occurrence and number of hospital admissions, type of hospital admission, length of hospital stay and inpatient death as recorded in the Western Australian Data Linkage System.

Results: Of 339 men with depressive symptoms, $152(44.8 \%)$ had at least 1 emergency hospital admission, compared with 1164 of $5072(22.9 \%)$ nondepressed men $(p<0.001)$. In multivariate analyses, the presence of depressive symptoms was a significant independent predictor of hospital admission (hazard ratio $1.67,95 \%$ confidence interval [CI] 1.38-2.01), number of hospital admissions (incidence rate ratio [IRR] 1.22, 95\% Cl 1.071.39) and total length of hospital stay (IRR 1.65, 95\% Cl 1.36-2.01).

Interpretation: Participants with depressive symptoms were at higher risk of hospital admission for nonpsychiatric conditions and were more likely to have longer hospital stays and worse hospital outcomes, compared with nondepressed participants. These results highlight the potential to target this high-risk group to reduce the burden of health care costs in an aging population.
Competing interests: Graeme J. Hankey has received funds from Boehringer Ingelheim for work on a Pradaxa advisory board and from Bayer for lectures on new anticoagulants in atrial fibrillation. No other competing interests declared.

This article has been peer reviewed.

Correspondence to: A. Matthew Prina, amp68@medschl.cam.ac.uk

CMAJ 2013. DOI:10.1503 /cmaj.121171
$\mathrm{O}$ lder people are the most frequent users of health services, and the progressive aging of the world's population may lead to a saturation of available services. Therefore, we must find ways to reduce preventable admissions to hospital and uncover the factors associated with potentially preventable use of health services. An association between depression and hospital admission for nonpsychiatric conditions has been postulated, although the data have been limited to specific clinical populations and the interpretation of the results hampered by the retrospective study design and the use of self-reported outcomes..$^{1-8}$ Consequently, these findings cannot be easily generalized or used to develop data-driven interventions.

We addressed this gap in the literature by using a community-based population survey with prospective data linkage to measure important health-related outcomes. Our main objective was to investigate whether community-dwelling older men with depressive symptoms were more likely than nondepressed men to be admitted to general hospitals. Our other aims were to determine whether the long-term clinical outcomes of these 2 groups differed in relation to the number of future hospital admissions, length of hospital stay and inpatient deaths.

\section{Methods}

\section{Patient sample}

We identified and selected participants from a community-derived sample of 5585 men living in Perth, Western Australia, who collectively compose the Health in Men Study cohort. The Health in Men Study is a prospective, follow-up study involving men aged 69 years and older who participated in an earlier trial of screening for abdominal aortic aneurysm. The full details of the cohort, including assessment procedures and enrolment are available elsewhere. ${ }^{9}$ In brief, 19352 men aged 65-83 years were randomly selected between Apr. 1, 1996, and Jan. 31, 1999, from the electoral roll (enrolment is mandatory for Australian citizens) and invited to participate in the study. A total of 12203 men completed the full questionnaire, which covered aspects of their lifestyle and medical history. The surviving men were invited to par- 
ticipate in a follow-up study between Oct. 1, 2001, and Aug. 31, 2004. The 4263 men who attended a face-to-face follow-up survey and the 1322 who completed a follow-up questionnaire constitute the Health in Men Study cohort $(n=5585)$. Both the face-to-face survey and the questionnaire included another health questionnaire and an assessment of mood status. All the participants who completed the mood assessment in the Health in Men Study (5411 [96.9\%]) were included in this analysis.

This study was approved by the Department of Health of Western Australia and the Human Research Ethics Committee of the University of Western Australia. All participants provided written informed consent.

Table 1: Baseline characteristics of 5411 men with valid 15-item Geriatric Depression Scale ratings, by depression status*

\begin{tabular}{|c|c|c|c|}
\hline \multirow[b]{2}{*}{ Characteristic } & \multicolumn{2}{|c|}{ Group, no. (\%) of participantst } & \multirow[b]{2}{*}{$p$ value } \\
\hline & $\begin{array}{c}\text { No depression } \\
n=5072\end{array}$ & $\begin{array}{c}\text { Depression } \\
n=339\end{array}$ & \\
\hline Age group, yr & & & $<0.001 \ddagger$ \\
\hline $69-74$ & $1804(35.6)$ & $85(25.1)$ & \\
\hline $75-79$ & $2185(43.1)$ & $150(44.2)$ & \\
\hline $80-84$ & $873(17.2)$ & $78(23.0)$ & \\
\hline$\geq 85$ & $208 \quad(4.1)$ & $26(7.7)$ & \\
\hline Missing values & $0 \quad(0.0)$ & $0 \quad(0.0)$ & \\
\hline Education & & & $<0.001 \ddagger$ \\
\hline None & $21 \quad(0.4)$ & $4 \quad(1.2)$ & \\
\hline Primary & $766(15.1)$ & $83(24.5)$ & \\
\hline Some secondary & 1915 (37.8) & $128(37.8)$ & \\
\hline Secondary & $1329(26.2)$ & $71(20.9)$ & \\
\hline Postsecondary & $1037(20.5)$ & $53(15.6)$ & \\
\hline Missing values & $2(0.0)$ & $0 \quad(0.0)$ & \\
\hline Smoking & & & $<0.001 \ddagger$ \\
\hline Never & $1682(33.2)$ & $69(20.4)$ & \\
\hline Past & $3132(61.8)$ & $238(70.4)$ & \\
\hline Current & $253 \quad(5.0)$ & $31 \quad(9.2)$ & \\
\hline Missing values & $3 \quad(0.1)$ & $1 \quad(0.3)$ & \\
\hline \multicolumn{3}{|c|}{ Duke Social Support Index tertiles } & $<0.001 \ddagger$ \\
\hline Highest & $2259(44.7)$ & $30 \quad(9.0)$ & \\
\hline Middle & $1608(31.8)$ & $71(21.3)$ & \\
\hline Lowest & $1181(23.4)$ & $232(69.7)$ & \\
\hline Missing values & $22 \quad(0.4)$ & $6 \quad(1.8)$ & \\
\hline $\begin{array}{l}\text { Charlson index (weighted), } \\
\text { mean }(95 \% \mathrm{Cl})\end{array}$ & $\begin{array}{c}1.17 \\
(1.12-1.22)\end{array}$ & $\begin{array}{c}2.21 \\
(1.94-2.48)\end{array}$ & $<0.001 \S$ \\
\hline Missing values & $571(11.3)$ & $14(4.1)$ & \\
\hline \multicolumn{4}{|c|}{$\begin{array}{l}\text { Note: } \mathrm{Cl}=\text { confidence interval, GDS- } 15=15 \text {-item Geriatric Depression Scale. } \\
{ }^{*} \text { No clinically significant depression }=\text { GDS- } 15 \text { score }<7 \text {; clinically significant depression = } \\
\text { GDS- } 15 \text { score } \geq 7 \text {. } \\
\text { †Unless stated otherwise. } \\
\neq \chi^{2} \text { test. } \\
\S \text { Student } t \text { test. }\end{array}$} \\
\hline
\end{tabular}

\section{Data sources}

We obtained administrative hospital records from the Western Australian Data Linkage System, ${ }^{10}$ which is a complex multiset system for the creation, storage, update and retrieval of links between health- and welfare-related data. The system integrates records from the Western Australian cancer, death and hospital morbidity registers, as well as the Mental Health Information System. The hospital morbidity register records all admissions to private and public hospitals since 1980, including codes for multiple medical diagnoses, admission and hospital type, and length of stay. The proportion of invalid (false positives) and missed links (negatives) were both estimated to be $0.11 \% .^{10}$

\section{Measure of depression}

We used the 15-item Geriatric Depression Scale (GDS-15) to assess depressive symptoms. Participants who scored a total of 7 or more points were defined as having clinically significant depressive symptoms. The relatively high cut-off was chosen a priori to ensure high specificity for the diagnosis of depression. ${ }^{11}$ We used previously published data to group the severity of depressive symptoms as follows: no depression (GDS-15 total score of 0 ), questionable depression (score of 1-4), mild-to-moderate depression (score of 5-9) and severe depression (score of 10-15). ${ }^{11}$

\section{Other measures}

Education level was subdivided into categories and measured as the highest level of education completed: no schooling, primary school, some secondary school, completed secondary school, completed university or other postsecondary degree. To assess social support, we used the Duke Social Support Index, a validated scale that measures individuals' satisfaction with their network of relationships. ${ }^{12}$

We assessed smoking status by asking men whether they had ever smoked and whether they were still smoking at the time of assessment. Finally, we used the Charlson weighted index, ${ }^{13}$ a widely used measure, to assess the presence of substantial medical comorbidity in our sample. Information about co-occurring medical conditions during the 10 years before assessment for the Health in Men Study was derived from the Western Australian Data Linkage System for all participants. We followed the procedures described by Quan and colleagues ${ }^{14}$ for the coding of algorithms, and we used Stagg's Stata routine to calculate Charlson index scores.

\section{Outcomes measures}

We investigated hospital admission $(0=$ not admitted, 1 = admitted), number of hospital admissions, 
mean total length of stay across hospital admissions, median length of stay, type of admission (elective v. emergency), overnight admission and inpatient death. For all analyses, we included only emergency (not elective) admissions, because they are more indicative of acute health problems.

We retrieved participants' hospital records for 24 months following assessment for the Health in Men Study. We chose this follow-up period a priori because we assessed depression only once and because we wished to generate data that would be comparable to other studies. ${ }^{15-17}$

\section{Statistical analysis}

We identified potential confounding variables by comparing baseline characteristics of participants with and without depression. We analyzed 24-month outcomes separately for all admissions and overnight admissions, and we stratified outcomes by depression status. We reported $p$ values from $\chi^{2}$ tests for categorical variables. Student $t$ test was used to compare the number of hospital admissions among men with and without depression. We used Mann-Whitney tests to compare length of hospital stay.

We reported incidence rate ratios (IRRs) with 95\% confidence intervals (CIs) after performing zero-inflated negative binomial regressions ${ }^{18,19}$ to account for overdispersed count outcome variables (mean length of stay, total length of stay, number of hospital admissions) with excess zeros. We used the Vuong nonnested test to assess the fit of the models. ${ }^{20}$

We plotted adjusted Kaplan-Meier curves, together with log-ranked test results, to compare cumulative admission rates of men with and without depression. We included age group, education level, Duke Social Support Index tertiles, smoking status and weighted Charlson index in the models as confounding variables. We estimated hazard ratios (HRs) by performing Cox regressions after checking that the proportional hazard assumption held. Finally, to investigate the association between depression and high-cost use of health services, we used Poisson regression analysis to determine mutually adjusted prevalence ratios with $95 \%$ CIs.

\section{Results}

A total of $5411(96.9 \%)$ men provided valid GDS-15 ratings and were included in the analysis. The mean age of participants was 76.8 (standard deviation 3.7) years, and $339(6.3 \%)$ had a

\begin{tabular}{|c|c|c|c|}
\hline \multirow[b]{2}{*}{ Outcome } & \multicolumn{2}{|c|}{ Group, mean $(95 \% \mathrm{Cl}) \dagger$} & \multirow[b]{2}{*}{$p$ value } \\
\hline & $\begin{array}{l}\text { No depression } \\
\quad n=5072\end{array}$ & $\begin{array}{l}\text { Depression } \\
\quad n=339\end{array}$ & \\
\hline $\begin{array}{l}\text { Emergency admission, } \\
\text { no. (\%) of participants }\end{array}$ & & & $<0.001$ \\
\hline No & $3908(77.0)$ & $187(55.2)$ & \\
\hline Yes & $1164(22.9)$ & $152(44.8)$ & \\
\hline No. of emergency admissions & $0.4(0.4-0.4)$ & $1.0(0.8-1.2)$ & $<0.001$ \\
\hline Total days in hospital & $11.9(10.9-12.9)$ & $21.0(15.9-26.0)$ & $<0.001$ \\
\hline Length of stay, $d$ & & & 0.009 \\
\hline$<2$ & $382(32.8)$ & $43(28.3)$ & \\
\hline $3-5$ & $360(30.9)$ & $33(21.7)$ & \\
\hline $6-12$ & $281(24.1)$ & $49(32.2)$ & \\
\hline$\geq 12$ & $141(12.1)$ & $27(17.8)$ & \\
\hline $\begin{array}{l}\text { Length of stay, } d \text {, median } \\
\text { (IQR) }\end{array}$ & $4.0(2.0-8.0)$ & $5.8(2.4-11.0)$ & 0.002 \\
\hline \multicolumn{4}{|c|}{$\begin{array}{l}\text { Note: } \mathrm{Cl}=\text { confidence interval, GDS- } 15=15 \text {-item Geriatric Depression Scale, } \\
\text { IQR }=\text { interquartile range. } \\
\text { *No clinically significant depression = GDS- } 15 \text { score }<7 \text {; clinically significant depression = } \\
\text { GDS- } 15 \text { score } \geq 7 \text {. } \\
\text { tUnless stated otherwise. }\end{array}$} \\
\hline
\end{tabular}

Table 3: Univariate and multivariate effects of depression on 2-year outcomes

\begin{tabular}{|c|c|c|c|c|c|c|}
\hline \multirow[b]{2}{*}{ Variable } & \multicolumn{2}{|c|}{$\begin{array}{l}\text { Mean length of stay, } \\
\text { IRR }(95 \% \mathrm{CI})^{*}\end{array}$} & \multicolumn{2}{|c|}{$\begin{array}{l}\text { Total length of stay, } \\
\text { IRR }(95 \% \mathrm{CI})\end{array}$} & \multicolumn{2}{|c|}{$\begin{array}{l}\text { No. of hospital admissions, } \\
\text { IRR }(95 \% \mathrm{CI})\end{array}$} \\
\hline & Univariate & Multivariate & Univariate & Multivariate & Univariate & Multivariate \\
\hline Depressiont & $1.32(1.13-1.53)$ & $1.25(1.06-1.48)$ & $1.76(1.47-2.12)$ & $1.65(1.36-2.01)$ & $1.30(1.15-1.47)$ & $1.22(1.07-1.39)$ \\
\hline Age & & $1.16(1.10-1.23)$ & & $1.27(1.18-1.36)$ & & $1.07(1.02-1.13)$ \\
\hline Education level & & $0.99(0.94-1.04)$ & & $0.96(0.91-1.02)$ & & $0.96(0.92-1.01)$ \\
\hline $\begin{array}{l}\text { Duke Social Support Index } \\
\text { tertiles }\end{array}$ & & $0.98(0.92-1.04)$ & & $1.01(0.94-1.09)$ & & $1.01(0.96-1.07)$ \\
\hline Smoking & & $0.94(0.84-1.07)$ & & $0.82(0.72-0.94)$ & & $0.88(0.80-0.98)$ \\
\hline Charlson index (weighted) & & $1.02(0.99-1.04)$ & & $1.08(1.05-1.11)$ & & $1.05(1.03-1.07)$ \\
\hline
\end{tabular}


GDS-15 score of 7 or greater. Compared to men without depression, those with depression were older, less educated and more likely to be current smokers, and they had a higher number of comorbidities (Table 1).

At the end of the study period, there had been a total of 2426 emergency admissions, most of which (2170) involved overnight stays; there were 8283 elective admissions. Of the 339 men with depression, $152(44.8 \%)$ had at least 1 emergency admission, compared with $1164(22.9 \%)$ of the 5072 men without depression $\left(\chi^{2}=82.7, p<\right.$ 0.001). Men with depression had a twofold increase in the mean number of hospital admissions, and these lasted on average twice as long as

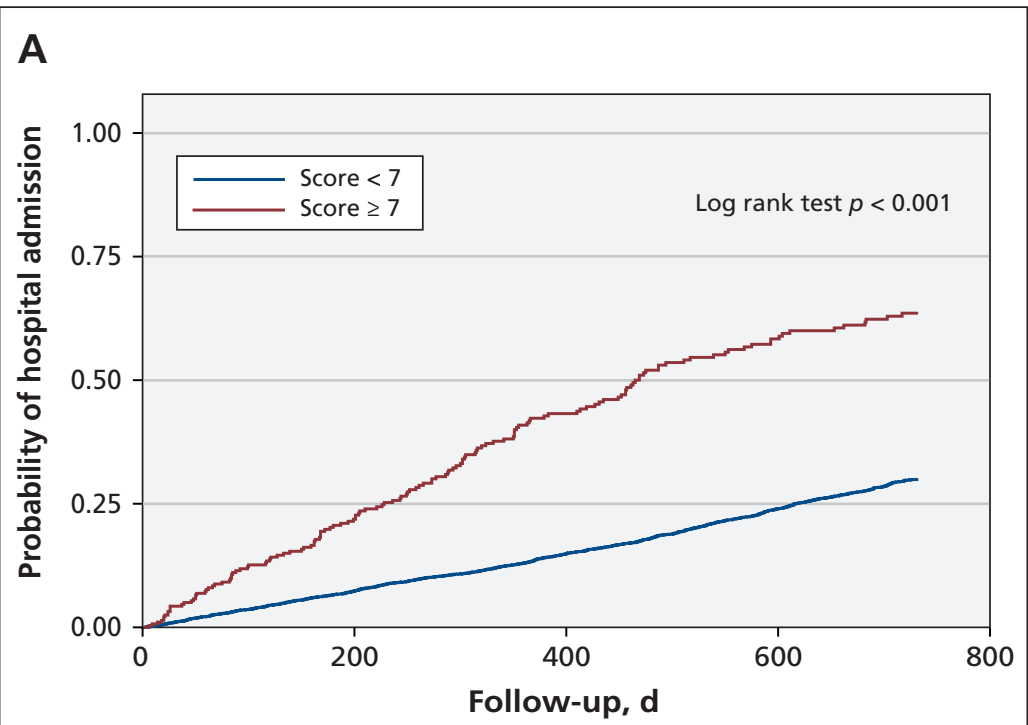

B

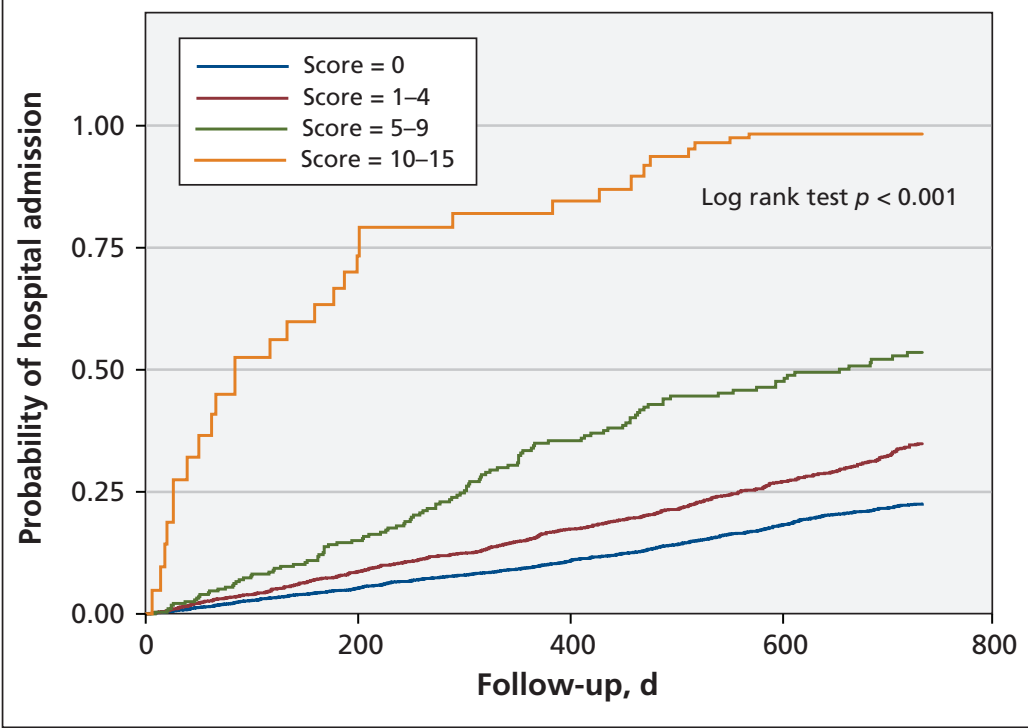

Figure 1: Kaplan-Meier estimates of hospital admissions by (A) depression status and (B) severity of symptoms. Adjusted for age, education level, smoking status, Duke Social Support Index tertiles and weighted Charlson index. Severity of symptoms: 15-item Geriatric Depression Scale score $=0$ (no depression), 1-4 (questionable depression), 5-9 (mild-to-moderate depression) and 10-15 (severe depression). for men without depression (Table 2). Overnight admissions were more frequent among men with depression (depression: 93.2\%; no depression: $\left.88.8 \% ; \chi^{2}=6.08, p=0.01\right)$ as were inpatient deaths (depression: 4.1\%; no depression: $1.5 \%$; $\left.\chi^{2}=19.82, p<0.001\right)$.

Length of stay and number of hospital admissions had very skewed distributions with an excess number of zeros. Potential confounders were therefore investigated by running zeroinflated negative binomial regressions (Table 3). Length of stay was longer and number of hospital admissions was higher among men with depressive symptoms compared with those without, even after adjustment. The adjusted IRR was 1.25 (95\% CI 1.06-1.48) for mean length of stay, 1.65 (95\% CI 1.36-2.01) for total length of stay and 1.22 (95\% CI 1.07-1.39) for number of hospital admissions. Vuong tests were significant, confirming that the use of zero-inflated models was indicated.

We investigated probability of hospital admission in the 2 groups by plotting Kaplan-Meier curves adjusted for age, education level, smoking status, Duke Social Support Index tertiles and comorbidities (Figure 1). We performed Cox regression analyses after computationally and graphically confirming the proportional hazards assumption (Table 4). In the fully adjusted model, the presence of depressive symptoms increased the hazard for hospital admission (HR 1.67 [95\% CI 1.38-2.01] and inpatient death (HR 1.81 [95\% CI 0.82-4.04]), though this was not statistically significant.

Increasing scores in the GDS-15 were also associated with higher HRs for both hospital admission and death, with men scoring 10 to 15 points at baseline having almost twofold higher HRs compared with men who scored 5 to 9 points (mild to moderate depression; Table 5).

A sensitivity analysis using a cut-off score of 5 points on the GDS-15 was also carried out. The findings were consistent and were not affected by this different cut-off (data not shown).

\section{Interpretation}

In this study, the presence of clinically significant symptoms of depression in older men was associated with increased risk of hospital admission, higher number of readmissions and longer use of services. These associations remained statistically significant after adjustment for several confounding variables.

Few studies have investigated the effect of clinically significant depressive symptoms on hospital admission and outcomes in people living in the community. A Danish group explored 
this association in a general population ${ }^{21}$ by following a group of 75-year-old adults over a 5year period. The group found a weak association between depression and subsequent hospital admission among women, and no association among men. This result is possibly explained by the relatively small sample size and the use of a depression scale not designed for older adults.

Wong and colleagues ${ }^{22}$ found a relation between depression and increased length of hospital stay and number of admissions in an older population in southern China, although the magnitude of the association was smaller than in our study. This may be because of a lower prevalence of depression at baseline and the use of self-report measures when recording comorbidities. Similar findings in diverse populations were described by von Ammon Cavanaugh and colleagues, ${ }^{23}$ who reported that a diagnosis of major depressive disorder and a history of depression independently predicted inpatient death. Finally, in a similar study by Prina and colleagues ${ }^{15}$ involving an older Dutch population, longer length of hospital stay and higher rates of admission and inpatient death were reported among depressed patients. However, only length of stay was associated with depression after adjustment for sociodemographic variables and comorbidities.

Several potential reasons can be proposed to explain the higher risk of hospital admission among older men with depression. Treatment adherence is known to be poor among patients with mood disorders. ${ }^{24}$ This could result in patients arriving in hospital at more acute or severe stages of their illness, potentially increasing length of stay and risk of death during admission. Our data show a higher number of emergency admissions than elective admissions among participants with depression, which is consistent with this hypothesis. Depression is also an inter-

Table 4: Cox regression analyses with dichotomous depression variable

\begin{tabular}{|lccccc}
\hline & \multicolumn{2}{c}{ Hospital admissions, HR $(95 \% \mathrm{Cl})$} & & \multicolumn{2}{c}{ Inpatient death, $\mathrm{HR}(95 \% \mathrm{Cl})$} \\
\cline { 2 - 3 } Variable & Univariate & Multivariate & & Univariate & Multivariate \\
\hline Depression* & $2.46(2.08-2.92)$ & $1.67(1.38-2.01)$ & & $3.82(1.85-7.9)$ & $1.81(0.82-4.04)$ \\
\hline Age & & $1.34(1.25-1.43)$ & & $2.42(1.78-3.31)$ \\
\hline Education level & $0.91(0.86-0.97)$ & & $1.14(0.87-1.50)$ \\
\hline $\begin{array}{l}\text { Duke Social Support Index } \\
\text { tertiles }\end{array}$ & $0.97(0.90-1.04)$ & & $0.84(0.58-1.20)$ \\
\hline Smoking & $0.76(0.67-0.86)$ & & $0.56(0.29-1.19)$ \\
\hline Charlson index (weighted) & $1.17(1.14-1.20)$ & & $1.19(1.08-1.25)$ \\
\hline
\end{tabular}

Note: $\mathrm{Cl}=$ confidence interval, GDS-15 = 15-item Geriatric Depression Scale, HR = hazard ratio.

*No clinically significant depression = GDS-15 score $<7$; clinically significant depression = GDS-15 score $\geq 7$.

Table 5: Cox regression analyses with grouped depression variable

\begin{tabular}{|c|c|c|c|c|}
\hline \multirow[b]{2}{*}{ Variable } & \multicolumn{2}{|c|}{ Hospital admissions, HR $(95 \% \mathrm{Cl})$} & \multicolumn{2}{|c|}{ Inpatient death, HR (95\% Cl) } \\
\hline & Univariate & Multivariate & Univariate & Multivariate \\
\hline \multicolumn{5}{|l|}{ Depression*† } \\
\hline GDS-15 score $=1-4$ & $2.06(1.83-2.31)$ & $1.70(1.50-1.92)$ & $3.72(2.0-6.89)$ & $2.45(1.26-4.75)$ \\
\hline GDS-15 score $=5-9$ & $2.98(2.45-3.61)$ & $2.08(1.68-2.58)$ & $6.11(2.53-14.8)$ & $3.05(1.15-8.13)$ \\
\hline GDS-15 score $=10-15$ & $4.66(3.23-6.72)$ & $3.06(2.10-4.46)$ & $11.00(2.54-47.7)$ & $4.38(0.89-21.4)$ \\
\hline Age & & $1.29(1.21-1.38)$ & & $2.28(1.67-3.12)$ \\
\hline Education level & & $0.93(0.87-0.98)$ & & $1.17(0.88-1.54)$ \\
\hline $\begin{array}{l}\text { Duke Social Support Index } \\
\text { tertiles }\end{array}$ & & $1.03(0.96-1.10)$ & & $0.93(0.65-1.33)$ \\
\hline Smoking & & $0.78(0.69-0.89)$ & & $0.60(0.30-1.22)$ \\
\hline Charlson index (weighted) & & $1.15(1.13-1.18)$ & & $1.16(1.05-1.29)$ \\
\hline
\end{tabular}


nalizing disorder that could potentially hamper effective communication with health care professionals, delaying a potential diagnosis and consequent treatment. Depressive symptoms in older adults could aggravate chronic diseases and disability. ${ }^{25}$ This could unfavourably influence older people's ability to look after themselves, leading to poorer self-perceived health, an increase in unexplained physical symptoms and, consequently, a rise in medical admissions. Furthermore, there is an association between the number of physical conditions and depression, and a dose-response relation has been described. ${ }^{26}$

In the current study, we have found that, even after adjustment for a robust measure of comorbidity (Charlson index), depression was a strong independent risk factor for hospital admission, longer hospital stays and worse hospital outcomes. This suggests that the association between depression and comorbidity, disability and hospital admission is complex and cannot be attributed solely to age, prevalent clinical morbidity, social support, education or smoking. However, even after adjustment for comorbidities, it is difficult to know to what extent depression may be a manifestation of early stages of diseases. We cannot therefore exclude the possibility that the findings may partially reflect depression as an epiphenomenon for other diseases.

We found a dose-response relation between depression severity and hospital admission, which suggests that reducing the symptoms may potentially improve hospital outcomes. However, subthreshold symptoms should not be underestimated, because they still have an impact on hospital admission and associated outcomes.

\section{Limitations}

Our study population was limited to men aged 69 years and older. We do not know whether our findings are generalizable to younger adults, women and people living outside Australia, although there is no obvious reason that this would not be the case, particularly in other developed countries. Although the GDS-15 has been proven to be a valid instrument for screening for major depressive disorder, it does not have the potential to differentiate between symptoms of major depressive disorder and depressive symptoms caused by other psychiatric diagnoses (e.g., dementia, psychosis), which could affect the interpretation of our results. We measured depressive symptoms only at baseline, and this exposure could have changed during the followup period. Hence, we were unable to determine whether change in depressive status could affect hospital outcomes.

Finally, the Charlson weighted index was originally created to estimate death and may not take into account all of the diagnoses that may increase hospital admission. Future research could involve a more comprehensive index to account for comorbidities.

\section{Conclusion}

Our study emphasizes the independent association between the presence of depressive symptoms in older men living in the community and hospital admissions, highlighting a possible target to identify men with potentially preventable admissions. Larger studies may be able to investigate effect modification, to determine more clearly what factors, if any, mediate the relation between depression and hospital outcomes. It is not clear whether reducing depressive symptoms would result in fewer hospital admissions, and further research is required to clarify this issue. Our data extend previous findings on the association between depression and hospital admission, with focus on the general population and admission frequency, length of stay and outcomes.

\section{References}

1. Rogal SS, Landsittel D, Surman O, et al. Pretransplant depression, antidepressant use, and outcomes of orthotopic liver transplantation. Liver Transpl 2011;17:251-60.

2. Sheeran T, Byers AL, Bruce ML. Depression and increased short-term hospitalization risk among geriatric patients receiving home health care services. Psychiatr Serv 2010;61:78-80.

3. Volz A, Schmid JP, Zwahlen M, et al. Predictors of readmission and health related quality of life in patients with chronic heart failure: a comparison of different psychosocial aspects. J Behav Med 2011;34:13-22

4. Turner A, Phillips L, Hambridge JA, et al. Clinical outcomes associated with depression, anxiety and social support among cardiac rehabilitation attendees. Aust N Z J Psychiatry 2010;44: 658-66.

5. Blanchette CM, Simoni-Wastila L, Shaya F, et al. Health care use in depressed, elderly, cardiac patients and the effect of antidepressant use. Am J Health Syst Pharm 2009;66:366-72.

6. Kronish IM, Rieckmann N, Schwartz JE, et al. Is depression after an acute coronary syndrome simply a marker of known prognostic factors for mortality? Psychosom Med 2009;71:697-703.

7. Kurdyak PA, Gnam WH, Goering P, et al. The relationship between depressive symptoms, health service consumption, and prognosis after acute myocardial infarction: a prospective cohort study. BMC Health Serv Res 2008;8:200.

8. Song EK, Lennie TA, Moser DK. Depressive symptoms increase risk of rehospitalisation in heart failure patients with preserved systolic function. J Clin Nurs 2009;18:1871-7.

9. Norman PE, Flicker L, Almeida OP, et al. Cohort profile: the Health In Men Study (HIMS). Int J Epidemiol 2009;38:48-52.

10. Holman CD, Bass AJ, Rouse IL, et al. Population-based linkage of health records in Western Australia: development of a health services research linked database. Aust N Z J Public Health 1999;23:453-9.

11. Almeida OP, Almeida SA. Short versions of the geriatric depression scale: a study of their validity for the diagnosis of a major depressive episode according to ICD-10 and DSM-IV. Int J Geriatr Psychiatry 1999;14:858-65.

12. Koenig HG, Westlund RE, George LK, et al. Abbreviating the Duke Social Support Index for use in chronically ill elderly individuals. Psychosomatics 1993;34:61-9.

13. Charlson ME, Pompei P, Ales KL, et al. A new method of classifying prognostic comorbidity in longitudinal studies: development and validation. J Chronic Dis 1987;40:373-83.

14. Quan H, Sundararajan V, Halfon P, et al. Coding algorithms for defining comorbidities in ICD-9-CM and ICD-10 administrative data. Med Care 2005;43:1130-9.

15. Prina AM, Deeg DJ, Brayne C, et al. The association between 
depressive symptoms and non-psychiatric hospitalisation in older adults. PLOS ONE 2012;7:e34821.

16. Kato N, Kinugawa K, Yao A, et al. Relationship of depressive symptoms with hospitalization and death in Japanese patients with heart failure. $J$ Card Fail 2009; 15:912-9.

17. Gudmundsson G, Gislason T, Janson C, et al. Risk factors for rehospitalisation in COPD: role of health status, anxiety and depression. Eur Respir J 2005;26:414-9.

18. Greene WH. Accounting for excess zeros and sample selection in poisson and negative binomial regression models: New York (NY): Stern School of Business, New York University; 1994.

19. Afifi AA, Kotlerman JB, Ettner SL, et al. Methods for improving regression analysis for skewed continuous or counted responses. Annu Rev Public Health 2007;28:95-111.

20. Vuong Q. Likelihood ratio tests for model selection and non-nested hypotheses. Econometrica 1989; 57:307-34.

21. Larsen K, Schroll M, Avlund K. Depressive symptomatology at age 75 and subsequent use of health and social services. Arch Gerontol Geriatr 2006;42: 125-39.

22. Wong SY, Mercer SM, Leung J, et al. The relationship between clinically relevant depressive symptoms and episodes and duration of all cause hospitalization in Southern Chinese elderly. J Affect Disord 2009; 113:272-8

23. von Ammon Cavanaugh S, Furlanetto LM, Creech $\mathrm{SD}$, et al. Medical illness, past depression, and present depression: a predictive triad for in-hospital mortality. Am J Psychiatry 2001;158:43-8.

24. Katon WJ. Epidemiology and treatment of depression in patients with chronic medical illness. Dialogues Clin Neurosci 2011;13:7-23.

25. Katon W. The impact of major depression on chronic medical illness. Gen Hosp Psychiatry 1996;18:215-9.

26. Wong SY, Mercer SW, Woo J, et al. The influence of multi-morbidity and self-reported socio-economic standing on the prevalence of depression in an elderly Hong Kong population. BMC Public Health 2008;8:119.

Affiliations: From the Department of Public Health and Primary Care (Prina, Brayne), Cambridge Institute of Public Health, Cambridge University, Cambridge, UK; the Western Australia Centre for Health and Ageing (Prina, Hankey, Flicker, Almeida), Centre for Medical Research, University of Western Australia; the School of Psychiatry and Clinical Neurosciences (Almeida), University of Western Australia, Crawley, Australia; the Department of Psychiatry (Almeida), Royal Perth Hospital, Perth, Australia; the Department of Neurology (Hankey), Royal Perth Hospital, Perth, Australia; the School of Medicine and Pharmacology (Yeap, Flicker), University of Western Australia, Crawley, Australia; the Department of Endocrinology and Diabetes (Yeap), Fremantle Hospital, Fremantle, Western Australia; the Department of Geriatric Medicine (Flicker), Royal Perth Hospital, Perth, Australia; the Departments of Epidemiology and Biostatistics, and of Sociology, and the EMGO Institute for Health and Care Research (Huisman), VU University, Amsterdam, The Netherlands.

Contributors: The study was designed by A. Matthew Prina, Martijn Huisman, Carol Brayne and Osvaldo Almeida. The analyses were conducted by A. Matthew Prina, who drafted the article. All of the authors interpreted the data and revised the manuscript. All of the authors approved the final version submitted for publication.

Acknowledgement: A. Matthew Prina was supported by an Endeavour Research Fellowship. No other specific funding was obtained for this project. 